\title{
Physical Activity Level and Adult Saudi Health Related Quality of Life
}

\author{
Salwa B. El-Sobkey \\ Department of Rehabilitation Health Sciences, College of Applied Medical Sciences, King Saud University, \\ Riyadh, Saudi Arabia \\ Email: salwa-el-sobkey@hotmail.com
}

Received 20 June 2014; revised 22 July 2014; accepted 5 August 2014

Copyright (C) 2014 by author and Scientific Research Publishing Inc. This work is licensed under the Creative Commons Attribution International License (CC BY). http://creativecommons.org/licenses/by/4.0/

(c) (i) Open Access

\begin{abstract}
Purpose: Physical inactivity in Saudi Arabia is a growing challenge to public health. This study aimed to define the relationship between the physical activity level (PAL) and adult Saudi healthrelated quality of life (HRQOL). Methods: Participants of this study were 100 Saudi adult aged from 18 - 70 years. The 36-Item Short-Form Health Status Survey (SF-36) was used to measure the participant's HRQoL. The SF-36 is comprised of 8 domains. The electronic version of the General Practice Physical Activity Questionnaire was used to categorize participants in terms of physical activity level into; inactive, moderately inactive, moderately active, and active. Descriptive and inferential statistical tests were used to treat the collected data. Results: Results showed that only $17 \%$ of the Saudi adults had active PAL. The PAL had significantly positive relationship with 4 domains of HRQoL including: physical functioning $(P=\mathbf{0 . 0 0 4})$, role limitations caused by physical health problems $(P=0.048)$, vitality $(P=0.009)$, and general health $(P=0.044)$. Results also confirmed that the younger the subject, the higher the PAL $(P=0.0001)$ and the better the HRQoL. This is true for all domains except the role limitations caused by emotional problems and emotional wellbeing. Conclusion: The poor physical activity level of adult Saudi negatively impacts their HRQoL.
\end{abstract}

\section{Keywords}

Saudi Physical Activity Level, Saudi Health Related Quality of Life, SF-36 Survey, General Practice Physical Activity Questionnaire

\section{Introduction}

Physical activity (PA) is associated with reduced risk for serious health conditions as coronary heart disease, cancers, diabetes, and stroke [1]. Unfortunately physical inactivity in Saudi Arabia (SA) is a growing challenge 
to public health [2] as its prevalence in the Saudi society ranged from $43 \%$ to as high as $99 \%$ in certain segments of the population [3]. Physical inactivity in SA is a significant consequence of the socioeconomic development including lifestyle changes and increase in obesity [3]-[5]. General Practice of Physical Activity Questionnaire (GPPAQ) which was developed in 2002 by the London school of hygiene and tropical medicine is a valid and reliable simple tool for ranking subject's physical activity level [6]-[8]. The GPPAQ is composed of 7 self-explanatory questions that can be answered in 30 - 60 seconds and are referred to the amount and type of physical activity in the workplace, the number of hours spent on different activities and the usual walking pace [6]-[8] The GPPAQ is suitable for adult from 16 - 74 years and its electronic template [9] automatically calculates the subject's data into physical activity index which categorizes the subject's physical activity level (PAL) as active, moderately active, moderately inactive, or inactive [6]-[8]. Meanwhile Health Related Quality of Life (HRQoL) is an important concept in health research and can help to inform decisions on prevention and treatment of ill health [1]. Because the WHO defines health as a state of complete physical, mental and social well-being and not merely the absence of disease or infirmity [10], many of the HRQoL questionnaires are designed to be multidimensional with different domains that cover the physical, functional, social, and psychological aspects of health. The Medical Outcomes Study 36-Item Short-Form Health Status Survey (SF-36) is perhaps the most widely used general HRQoL survey instrument in the world today [11]. It was constructed to survey health status and was designed for use in clinical practice, research, and general population surveys [12]. It is comprised of 36 items that assessed 8 health concepts: physical functioning (PF) (10 items), role limitations caused by physical health problems (RLPHP) (4 items), role limitations caused by emotional problems (RLEP) (3 items), social functioning (SF) (2 items), emotional well-being (EW) (5 items), vitality (4 items), body pain (BP) (2 items), and general health (GH) (5 items) perceptions [11]-[13]. The SF-36 was constructed for self-administration by persons 14 years of age and older, and for administration by a trained interviewer in person or by telephone [12]. It has been validated and can be self-administered with reliability [14]. A better understanding of how healthy lifestyles, such as PA, can influence HRQoL which might help to inform policy makers intending to encourage PA in the general population [15]. In spite, evidence on the association between HRQoL and PA in the general population is limited as previous research has focused on specific interventions or populations with chronic conditions [16] or the long-term effects of sustained PA on the incidence of chronic conditions [1]. The current study aimed to address this gap of knowledge by defining the relationship between the adult Saudi PAL and their HRQoL and examine the hypothesis that higher PAL would be associated with better HRQoL.

\section{Method}

This cross sectional study was conducted in Riyadh, SA during the period from March to July, 2012. Participants were recruited from the community through verbal invitation and with assistant of the King Saud University students. Subjects who voluntary agreed to participate in the study were checked for eligibility. They should be adult Saudis aged 16 - 74 years, free from diagnosed health illness, and non smokers. They received full explanation of the aim and procedures of the study before they signed the study consent form. Out of the 500 subjects who agreed to participate in the study, 100 were matching the inclusion criteria. To define the PAL of the participants, researcher used the electronic template of the GPPAQ available on

http://www.patient.co.uk/doctor/general-practice-physical-activity-questionnaire-gppaq [9] with i-pad to record answers of the participant on the questionnaire's questions and the physical activity index was calculated electronically. The physical activity index categorized the participants PAL into active, moderately active, moderately inactive, and inactive.

Through the interview the eligible participants also answered Arabic version of the SF-36 survey [17]. Researcher recorded the participant's answers, scored them, and then calculated the percentage for the 8 domains measured by the survey. These domains included physical functioning (10 items), role limitations caused by physical health problems (4 items), role limitations caused by emotional problems ( 3 items), social functioning (2 items), emotional well-being (5 items), vitality (4 items), body pain (2 items), and general health (5 items). All items were scored so that high score defining a more favorable health state. In addition each item was scored on a 0 to 100 range so that the lowest and highest possible scores were set at 0 and 100 respectively [18].

\section{Statistical Data Analysis}

Collected data were treated statistically using SPSS version 21. For descriptive statistics mean and standard 
deviation (SD) were used for continuous variable (age) while frequency distribution was used for categorical variables (gender, PAL). Inferential statistics included Person correlations to define the relationships between the percentage values of the 8 domains of SF-36 and each of the participant's PAL, age and gender. Participants were divided into 3 age groups (18 - 39, 40 - 59, and 60 - 74 years). Chi-square tests were used to investigate the association between PAL and both gender and age categories. P value less than 0.05 was considered significant.

\section{Results}

The demographic characters (Table 1) of the current study participants showed that they were 100 adult Saudi subjects aged from 18 to 70 years (mean and SD are $39.6 \pm 17.07$ years) with 52\% females.

Only $17 \%$ of them had active PAL. Poor PAL had no significant association with gender $(\mathrm{P}=0.929)$ (Figure 1).

Meanwhile there was a significant association between PAL and age categories $(\mathrm{P}=0.0001)$ (Figure 2). The better PAL showed among younger adult Saudis was confirmed with significantly negative correlation between PAL and age $(\mathrm{N}=98, \mathrm{r}=-0.449, \mathrm{P}=0.0001)$.

The PAL was shown to be positively correlated with the adult Saudi HROoL domains. The correlated SF-36 survey domains were physical function $(\mathrm{P}=0.004)$, role limitation caused by physical health problem $(\mathrm{P}=$ 0.048), vitality $(\mathrm{P}=0.009)$, and general health $(\mathrm{P}=0.044)$ domains (Table 2$)$.

On the other hand age was negatively correlated to HRQoL. The younger the subject, the better the HRQoL and this was significantly applied to all domains of SF-36 survey except RLEP and EW domains (Table 3).

Table 1. Demographic characters of the participants $(\mathrm{N}=100)$.

\begin{tabular}{cccccc}
\hline & N & Minimum & Maximum & Mean & SD \\
\hline Age (year) & 100 & 18 & 70 & 39.6 & 17.07 \\
\hline \multirow{3}{*}{ Gender } & & Frequency & Percent & & \\
& Female & 52 & 52 & \\
& Male & 48 & 48 & \\
& Total & 100 & 100 & \\
Physical activity level & Inactive & 41 & 27 & 13 \\
& Moderately inactive & 27 & 17 & \\
& Moderately active & 13 & 17 & 100 \\
\hline
\end{tabular}

Table 2. The relationship between adult Saudi physical activity level and domains of health related quality of life measured with Short-From 36 Health Status Survey.

\begin{tabular}{|c|c|c|c|c|}
\hline & \multirow{2}{*}{ Domains of HRQoL } & \multicolumn{3}{|c|}{ Total } \\
\hline & & $\mathbf{N}$ & $\mathbf{r}$ & $\mathbf{P}$ \\
\hline \multirow{8}{*}{$\begin{array}{c}\text { Physical } \\
\text { Activity } \\
\text { Level }\end{array}$} & Physical Function & \multirow{8}{*}{98} & $0.291^{* *}$ & 0.004 \\
\hline & Role Limitation Caused by Physical Health Problem & & $0.200^{*}$ & 0.048 \\
\hline & Role Limitation Caused by Emotional Problem & & 0.059 & 0.566 \\
\hline & Social Function & & 0.059 & 0.350 \\
\hline & Emotional Well-Being & & -0.078 & 0.448 \\
\hline & Vitality & & $0.262^{* *}$ & 0.009 \\
\hline & Body Pain & & 0.159 & 0.117 \\
\hline & General Health & & $0.204^{*}$ & 0.044 \\
\hline
\end{tabular}

${ }^{*}$ Correlation is significant at the 0.05 level (2-tailed); ${ }^{* *}$ Correlation is significant at the 0.01 level (2-tailed). 
Table 3. The relationship between adult Saudi age and domains of health related quality of life measured with Short-From 36 Health Status Survey.

\begin{tabular}{|c|c|c|c|c|}
\hline & Domains of HRQoL & $\mathbf{N}$ & $\mathbf{r}$ & $\mathbf{P}$ \\
\hline \multirow{8}{*}{$\begin{array}{c}\text { Age } \\
18 \text { - } 70 \text { Years }\end{array}$} & Physical Function & \multirow{8}{*}{100} & $-0.559^{* *}$ & 0.0001 \\
\hline & Role Limitation Caused by Physical Health Problem & & $-0.417^{* *}$ & 0.0001 \\
\hline & Role Limitation Caused by Emotional Problem & & -0.153 & 0.130 \\
\hline & Social Function & & $-0.227^{*}$ & 0.023 \\
\hline & Emotional Well-Being & & -0.034 & 0.734 \\
\hline & Vitality & & $-0.268^{* *}$ & 0.007 \\
\hline & Body Pain & & $-0.428^{* *}$ & 0.0001 \\
\hline & General Health & & $-0.443^{* *}$ & 0.0001 \\
\hline
\end{tabular}

${ }^{*}$ Correlation is significant at the 0.05 level (2-tailed); ${ }^{* *}$ Correlation is significant at the 0.01 level (2-tailed).

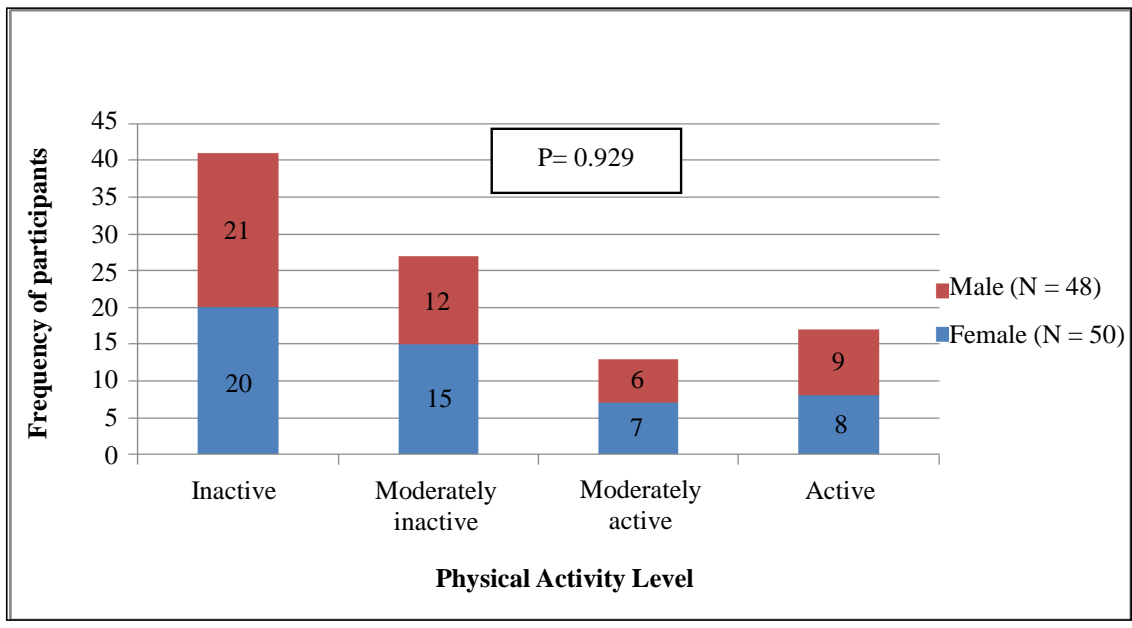

Figure 1. Assocation between adult Saudi physical activity level and gender $(\mathrm{N}=98)$.

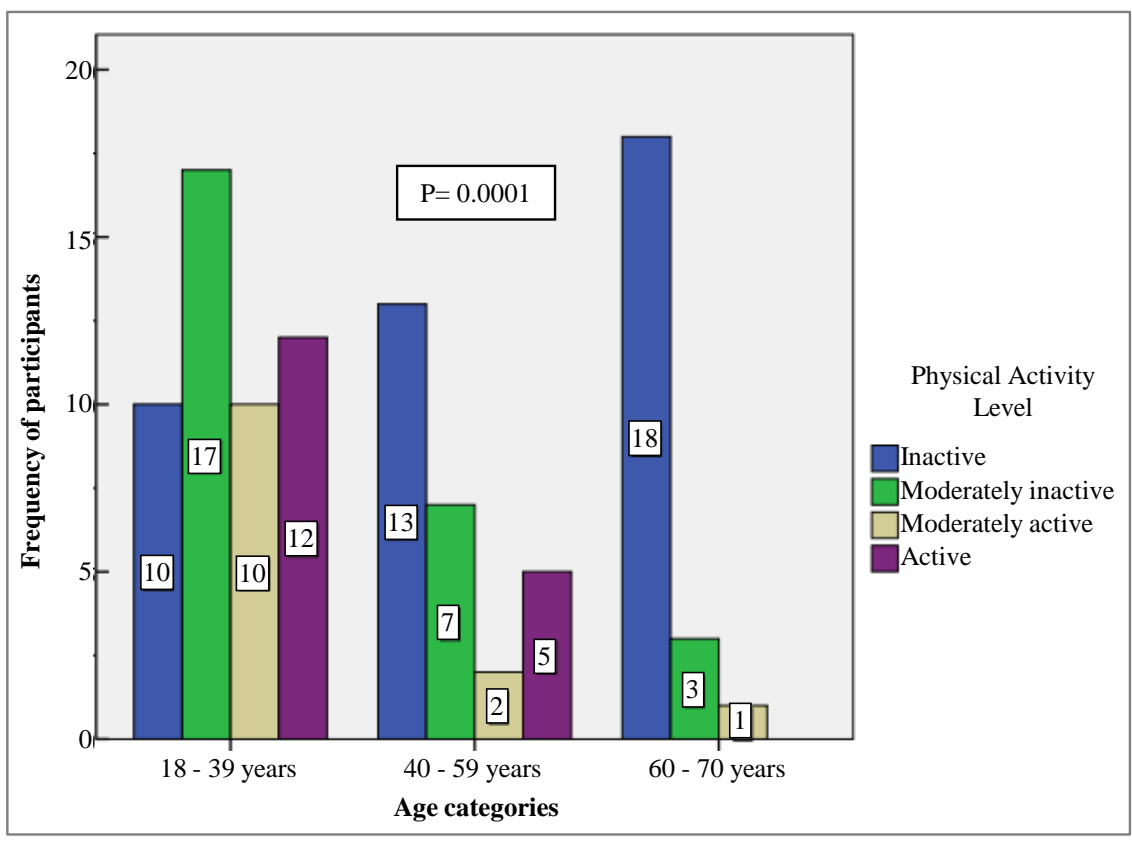

Figure 2. Physical activity level among different age categories of adult Saudi (N = 98). 


\section{Discussion}

The physical activity is one component of healthy life style. The high PAL promotes health and prevents illness. Risk of diseases increases with physical inactivity [1] [3]-[5]. Moreover, it has been estimated that physical inactivity accounts for $6 \%$ - 10\% of all global deaths annually [19]. During the last few decades, adult Saudi had enjoyed the modern Western civilization but at the same time had progressively suffered from its disadvantage; obesity and inactivity. Several Saudi studies reported increases in the percentage of adult Saudis with low PAL [2]-[5] [20]. Need to investigate the relationship between PAL and HRQoL was raised by the above mentioned facts. That is why the current study aimed to define the relationship between the PAL and adult Saudi HRQoL as well as to examine the hypothesis that higher PAL would be associated with better HRQoL.

\subsection{Saudi PAL}

The current findings, concurrent with previous studies, showed that low percentage (17\%) of the adult Saudis are active. Gender has nothing to do with the poor PA of adult Saudi. Both males and females are suffering from inactivity. This is because the underlying cause of inactivity, sedentary automated life style, is actually practiced by both males and females. Study findings of Al-Nozha et al. (2007) revealed that Saudi males are significantly more active than females [5]. The participants' age difference between their study and current study may explain the findings difference. In the current study the age was 18 - 70 years while in their study it was 30 - 70 years. The younger age of participants may eliminate the gender PAL difference. A second cause could be the difference in instruments used to record the participants' PAL. In the current study the GPPAQ was used which is consigned about the walking activity among other daily physical activities. On the other hand the questionnaire used by the Al-Nozha et al. (2007) including sport-related physical activities. Because of culture issues, Saudi males have more opportunity to practice sports activities than females. That is why they recorded higher PAL than females. On the other hand age showed to have significant inverse relation with PAL. Out of the 22 old persons (60 - 70 years) participated in the current study, 18 (81.8\%) of them are inactive. Adding age-related physiological changes to the sedentary life style could be the proper explanation for more inactivity among old persons. Aging is accompanied by progressive systemic physiological deterioration and consequently functional reduction.

\subsection{PAL and Saudi HRQoL}

Results supported the hypothesis that higher level of PA is associated with better HRQoL. This statistically proved hypothesis could be explained through physiological basis. The evidence regarding health benefits of PA is overwhelming and continues to accumulate since ancient Greek [21]-[23]. Regular PA has structural and functional beneficial effects on the cardiovascular system [24]. It guarantees the integrity of the endothelial function and vascular smooth muscle function, promotes the decrease of oxidative stress, decreases the sympathetic tone, enhances the parasympathetic autonomic tone and promotes the development of new muscular capillaries [22]-[25]. The PA also increases the maximal oxygen uptake, stroke volume, cardiac output, coronary artery function, the maximal systemic arteriovenous oxygen difference, cardiorespiratory fitness, and functional exercise capacity [26]-[29]. It positively influences most structural components of the musculoskeletal system [30]. The benefits of PA are not limited to the somatic physiological and functional aspects but it includes the psychological aspect as well. The psychological benefits associated with PA included; relaxation, increased social contact, promotion of self care, self efficacy, self esteem, promotion of positive mental health, psychological well being and cognitive functioning [31] [32]. These benefits of PA allow the physically active person to have physiological, functional, and psychological fitness and consequently higher HRQoL.

\subsection{HRQoL Domains Related to PAL among Saudis}

The current study showed that the most correlated domains of HRQoL, as measured with SF-36 survey, to the person's PAL were physical function, role limitation caused by physical health problem, vitality, and general health. These domains seem to be related more to the physiological functional aspects rather than the psychological one. Recall that the above mentioned physiological benefits of PA can help to understand how inactivity could cause reduction in the musculoskeletal and cardiopulmonary fitness. With no doubt reduced fitness would have direct negative impact on the person's physical function; role limitation caused by physical health problem, 
vitality, and consequently general health domains of HRQoL. We can also add that PA does not only positively influence the structural components of the musculoskeletal system that are related to functional capabilities and the risk of degenerative diseases, but PA also has the potential to postpone or prevent musculoskeletal disorders, such as mechanical low back pain, neck and shoulder pain, and osteoporosis and related fractures [30]. Of course this prevention function of the PA would guarantees better PF, RLPHP, Vitality, and GH for persons with higher PAL. Other populations had similar results as the Saudi population. Australian women aged from 50 - 80 years also showed negative curvilinear correlation between their PA, measured by the international physical activity questionnaire, and HRQoL, measured with SF-36, particularly the physical function and vitality domains [33].

Meanwhile we should not ignore that religious connectedness is common phenomenon in Saudi Arabia [34]. The high moral aspects of Saudi population are derived from their Islamic culture. Islamic principles include faith, satisfaction and moral calmness. These principles could positively affect the psychological aspects of adult Saudi and overcome any possible correlation between PAL and HRQoL domains related to psychological aspects, particularly the role limitation caused by emotional problem and emotional well-being. This hypothesis is emphasized by the study done by Alzahrani et al. (2013) as they found that there was a strong positive relationship between religious connectedness and Saudi HRQL [34]. Sarvimäki et al. (2000) defined the QoL as sense of well-being which includes coping mechanisms with 2 factors; intra-individual factor and external factors as biophysical and sociocultural environment [35]. Although the external factors could not be controlled by the persons, the intra-individual factor might be enhanced by the persons' religious concepts and so improve their ability to manage successful coping mechanisms, higher emotional well-being, and better HRQoL.

\subsection{Aging and Saudi HRQoL}

Current study's results revealed negative correlation between age and Saudi HRQoL. Echoing the age-related physiological changes can give reason why old Saudi had lower HRQoL than younger. Let us also not forget that the majority of Saudi were inactive. Aging physiological and pathological changes and low PAL is a combination causes Saudi to progressively have lower HRQoL as they get older. The negative correlation was true for all HRQoL domains except ROEP and EW. The hypothesis of Islamic culture and religious connectedness protection against psychological and emotional problems might be the proper explanation for this result.

\section{Limitation of the Study}

Opposite of the objective PAL tools (as pedometer), PAL questionnaires are dependent on participants' perceptions. Participants may over or under estimate their PAL. The present study is an association study and that does not imply any causation between the PAL and HRQoL or which one precedes the other. The participants of the current study were from a volunteer group and that those people who may dislike PA may have elected not to take part in this study.

\section{Conclusion}

Adult Saudi aged from 18 - 74 years had significantly positive relationship between their PAL and their HRQoL. The higher the PAL is, the better the HRQoL. This is particularly true for the HRQoL domains of the physical function, role limitation caused by physical health problem, vitality, and general health.

\section{Recommendation}

All effort should be made throughout the health professions to promote PA.

\section{References}

[1] Anokye, N., Trueman, P., Green, C., Pavey, T. and Taylor, R. (2012) Physical Activity and Health Related Quality of Life. BMC Public Health, 12, 624. http://dx.doi.org/10.1186/1471-2458-12-624

[2] Khalaf, A., Ekblom, Ö. and Al-Hazzaa, H. (2013) Female University Students’ Physical Activity Levels and Associated Factors-A Cross-Sectional Study in Southwestern Saudi Arabia. International Journal of Environmental Research and Public Health, 10, 3502-3517. http://dx.doi.org/10.3390/ijerph10083502

[3] Al-Hazzaa, H. (2010) Physical Inactivity in Saudi Arabia. An Underserved Public Health Issue. Saudi Medical Journal, 


\section{1, 1278-1279.}

[4] Khawaja, M., Dawns, J., Meyerson-Knox, S. and Yamout, R. (2008) Disparities in Child Health in the Arab Region during the 1990s. International Journal for Equity in Health, 7, 24. http://dx.doi.org/10.1186/1475-9276-7-24

[5] Al-Nozha, M., Al-Hazzaa, H., Arafah, M., Al-Khadra, A., Al-Mazrou, Y., Al-Maatouq, M., et al. (2007) Prevalence of Physical Activity and Inactivity among Saudis Aged 30-70 Years. A Population-Based Cross-Sectional Study. Saudi Medical Journal, 28, 559-568.

[6] Heron, N., Tully, M., McKinley, M. and Cupples, M. (2014) Physical Activity Assessment in Practice: A Mixed Methods Study of GPPAQ Use in Primary Care. BMC Family Practice, 15, 11-19. http://dx.doi.org/10.1186/1471-2296-15-11

[7] A Guide to General Practice Physical Activity Questionnaire. http://better.utoronto.ca/wp-content/uploads/2012/10/GPPAQ-guide.pdf

[8] General Practice Physical Activity Questionnaire. https://www.gov.uk/government/publications/general-practice-physical-activity-questionnaire-gppaq

[9] Electronic Template of General Practice Physical Activity Questionnaire. http://www.patient.co.uk/doctor/general-practice-physical-activity-questionnaire-gppaq

[10] World Health Organization Definition of Health. http://www.who.int/about/definition/en/print.html

[11] Hays, R. and Morales, L. (2001) The RAND-36 Measure of Health-Related Quality of Life. Annals of Medicine, 33, 350-357. http://dx.doi.org/10.3109/07853890109002089

[12] Ware, J. and Sherbourne, C. (1992) The MOS 36-Item Short-Form Health Survey (SF-36). I. Conceptual Framework and Item Selection. Medical Care, 30, 473-483. http://dx.doi.org/10.1097/00005650-199206000-00002

[13] Hays, R., Sherbourne, C. and Mazel, R. (1993) The RAND 36-Item Health Survey 1.0. Health Economics, 2, $217-227$. http://dx.doi.org/10.1002/hec.4730020305

[14] Patel, A., Donegan, D. and Albert, T. (2007) The 36-Item Short Form. Journal of the American Academy of Orthopaedic Surgeons, 15, 126-134.

[15] Chai, W., Nigg, C., Pagano, I., Motl, R., Horwath, C. and Dishman, R. (2010) Associations of Quality of Life with PA, Fruit and Vegetable Consumption, and Physical Inactivity in a Free Living, Multi-Ethnic Population in Hawaii: A Longitudinal Study. International Journal of Behavioral Nutrition and Physical Activity, 7, 83. http://dx.doi.org/10.1186/1479-5868-7-83

[16] Bize, R., Johnson, J. and Plotnikoff, R. (2007) Physical Activity Level and Health-Related Quality of Life in the General adult Population: A Systematic Review. Preventive Medicine, 45, 401-415. http://dx.doi.org/10.1016/j.ypmed.2007.07.017

[17] Al Abdulmohsin, S., Goons, S., Draugalis, J. and Hays, R. (1997) Translation of the RAND 36-Item Health Survey 1.0 (aka SF-36) into Arabic. RAND, 7995-8020.

[18] How to Score SF-36 Questionnaire. http://www.chiro.org/LINKS/OUTCOME/How to score the SF-36.pdf

[19] Lee, I., Shiroma, E., Lobelo, F., Puska, P., Blair, S. and Katzmarzyk, P. (2012) Effect of Physical Inactivity on Major Non-Communicable Diseases Worldwide: An Analysis of Burden of Disease and Life Expectancy. Lancet, 380, 219229. http://dx.doi.org/10.1016/S0140-6736(12)61031-9

[20] Al-Eisa, E. and Al-Sobayel, H. (2012) Physical Activity and Health Beliefs among Saudi Women. Journal of Nutrition and Metabolism, 2012, Article ID: 642187.

[21] González, G. and Sedentarism, M. (2013) Active Lifestyle and Sport: Impact on Health and Obesity Prevention. Nutrición Hospitalaria, 28, 89-98.

[22] Adamu, B., Sani, M. and Abdu, A. (2006) Physical Exercise and Health: A Review. Nigerian Journal of Medicine, 15, 190-196.

[23] Prasad, D. and Das, B. (2009) Physical Inactivity: A Cardiovascular Risk Factor. Indian Journal of Medical Sciences, 63, 33-42. http://dx.doi.org/10.4103/0019-5359.49082

[24] Conti, A. and Macchi, C. (2013) Protective Effects of Regular Physical Activity on Human Vascular System. La Clinica Terapeutica, 164, 293-294.

[25] Leung, F., Yung, L., Laher, I., Yao, X., Chen, Z. and Huang, Y. (2008) Exercise, Vascular Wall and Cardiovascular Diseases: An Update (Part 1). Sports Medicine, 38, 1009-1024. http://dx.doi.org/10.2165/00007256-200838120-00005

[26] Blomqvist, C. (1983) Cardiovascular Adaptation to Physical Training. Annual Review of Physiology, 45, 169-189. http://dx.doi.org/10.1146/annurev.ph.45.030183.001125

[27] Mutikainen, S., Perhonen, M., Alén, M., Leskinen, T., Karjalainen, J., Rantanen, T., Kaprio, J. and Kujala, U.M. (2009) Effects of Long-Term Physical Activity on Cardiac Structure and Function: A Twin Study. Journal of Sports Science 
\& Medicine, 8, 533-542.

[28] Hägg, U., Wandt, B., Bergström, G., Volkmann, R. and Gan, L. (2005) Physical Exercise Capacity Is Associated with Coronary and Peripheral Vascular Function in Healthy Young Adults. American Journal of Physiology-Heart and Circulatory Physiology, 289, 1627-1634. http://dx.doi.org/10.1152/ajpheart.00135.2005

[29] Cheng, Y., Macera, C., Addy, C., Sy, F., Wieland, D. and Blair, S. (2003) Effects of Physical Activity on Exercise Tests and Respiratory Function. British Journal of Sports Medicine, 37, 521-528. http://dx.doi.org/10.1136/bjsm.37.6.521

[30] Vuori, I. (1995) Exercise and Physical Health: Musculoskeletal Health and Functional Capabilities. Research Quarterly for Exercise and Sport, 66, 276-285. http://dx.doi.org/10.1080/02701367.1995.10607912

[31] Scully, D., Kremer, J., Meade, M., Graham, R. and Dudgeon, K. (1998) Physical Exercise and Psychological Well Being: Acritical Review. British Journal of Sports Medicine, 32, 111-120. http://dx.doi.org/10.1136/bjsm.32.2.111

[32] Martinsen, E. (1995) Effect of Exercise on Mental Health in Clinical Populations. In: Biddle, S.J.H., Ed., European Perspectives on Exercise and Sport Psychology, Human Kinetics, Champaign, 71-90.

[33] Heesch, K., Uffelen, J., Gellecum, Y. and Brown, W. (2012) Dose-Response Relationships between Physical Activity, walking and Health-Related Quality of Life in Mid-Age and Older Women. Journal of Epidemiology and Community Health, 66, 670-677. http://dx.doi.org/10.1136/jech-2011-200850

[34] Alzahrani, H. and Sehlo, M. (2013) The Impact of Religious Connectedness on Health-Related Quality of Life in Patients with Diabetic Foot Ulcers. Journal of Religion and Health, 52, 840-850. http://dx.doi.org/10.1007/s10943-011-9529-X

[35] Sarvimäki, A. and Stenbock-Hult, B. (2000) Quality of Life in Old Age Described as a Sense of Well-Being, Meaning and Value. Journal of Advanced Nursing, 32, 1025-1033. http://dx.doi.org/10.1046/j.1365-2648.2000.01568.x 
Scientific Research Publishing (SCIRP) is one of the largest Open Access journal publishers. It is currently publishing more than 200 open access, online, peer-reviewed journals covering a wide range of academic disciplines. SCIRP serves the worldwide academic communities and contributes to the progress and application of science with its publication.

Other selected journals from SCIRP are listed as below. Submit your manuscript to us via either submit@scirp.org or Online Submission Portal.
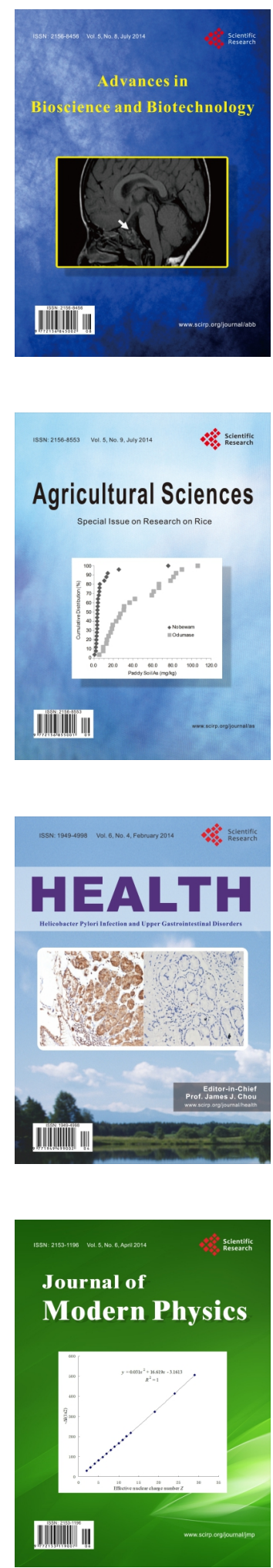
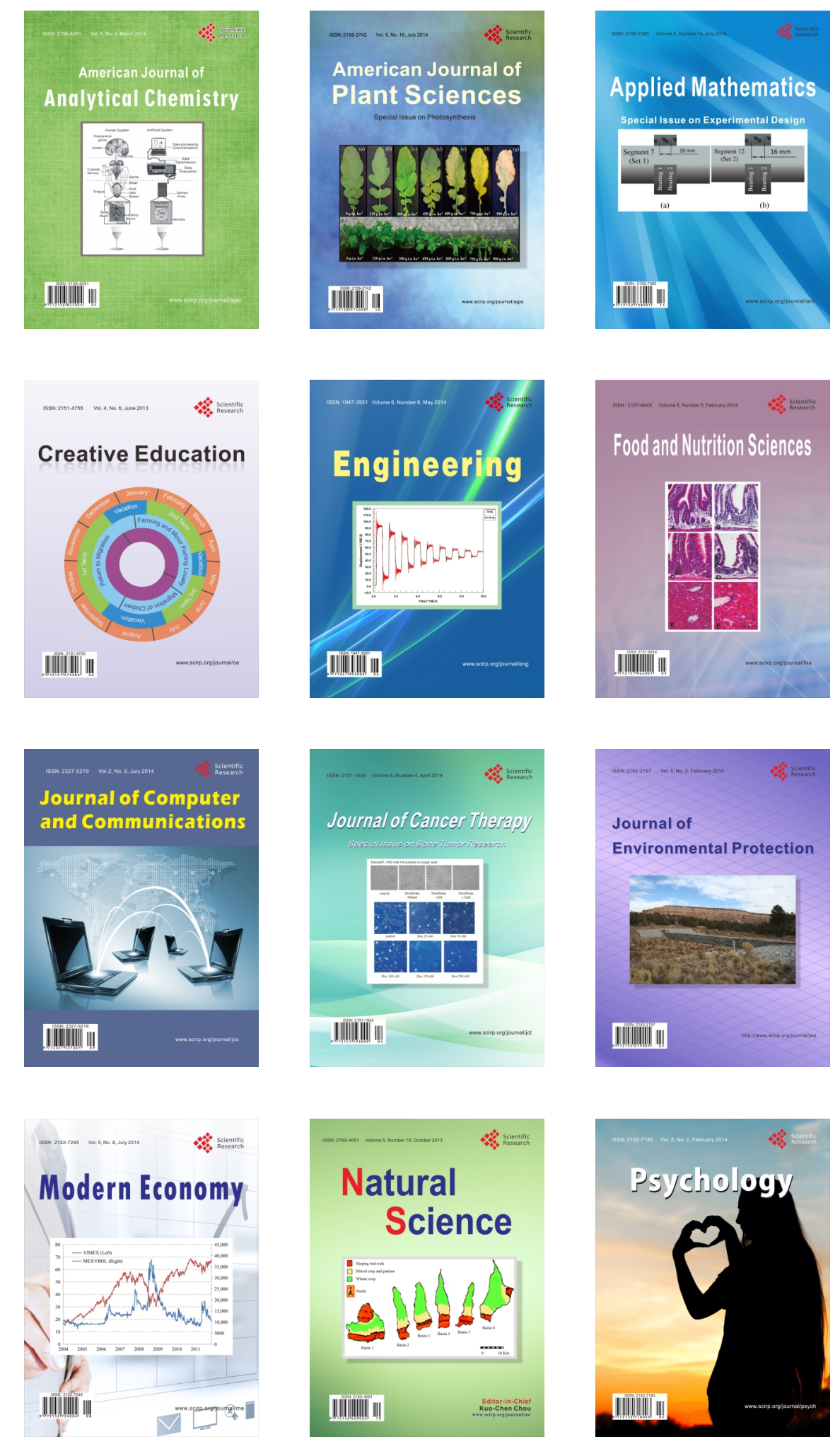